\title{
Resin directional flow and degree of cure sensing using chirped optical fibre long period gratings
}

\author{
Rebecca Y N Wong, Edmon Chehura, Stephen W James, and Ralph P Tatam*, Fellow, InstP and SPIE
}

\begin{abstract}
The use of chirped long period gratings (CLPGs) for monitoring the flow, the direction of the flow and the subsequent cure of an epoxy resin is presented. The asymmetric properties of the CLPG and its sensitivity to refractive index changes were exploited to facilitate the measurement of the direction of the flow. The performances of a continuously chirped LPG (CCLPG) and a step chirped LPG (SCLPG) were compared, with the CCLPG showing improved spatial resolution. The CCLPG and SCLPG were also used to monitor changes in the refractive index of the resin during cure, with the results showing close agreement with measurements undertaken simultaneously using a fibre optic Fresnel refractometer.
\end{abstract}

Index Terms - Fibre optics, optical fibre sensor, long period grating, chirped grating, directional flow, resin cure, Fresnel sensor

\section{INTRODUCTION}

$\mathrm{T}$ HE increasing use of composite materials in the aerospace, automotive and marine industries can be attributed to their high strength-to-weight ratio, long lifetime and high corrosion resistance [1].There is a requirement for sensors to monitor all stages of manufacture of composite materials, in particular the infusion of the resin into the preform and its subsequent cure. The monitoring of infusion and the degree of cure of the resin are currently carried out empirically [2], as pre-production measurement tools such as differential scanning calorimetry [3] are not suitable for online process monitoring, while dielectric sensors [4] are not suited to being embedded into carbon fibre reinforced composites.

The low permeability of a fibre reinforced preform provides resistance to resin flow, which can lead to inconsistencies when the resin is not uniformly spread throughout the material

Manuscript submitted April 3, 2017. This work was supported by the United Kingdom Engineering and Physical Sciences Research Council (EPSRC) under Grant EP/H02252X/1

E. Chehura, S. W. James and R. P. Tatam are with the Engineering Photonics, Cranfield University, Cranfield, MK43 0AL, United Kingdom (email: $\quad$ e.chehura@cranfield.ac.uk; s.w.james@cranfield.ac.uk; r.p.tatam@cranfield.ac.uk)

R. Y. N. Wong was with the Engineering Photonics, Cranfield University, Cranfield, MK43 OAL, United Kingdom. She is now with the Smart Energy and Environment Cluster, Institute for Infocomm Research, 1 Fusionopolis Way, \#21-01 Connexis (South Tower), Singapore 138632 (e-mail: rebeccawong@i2r.a-star.edu.sg).
[5]. When this occurs, the structural integrity of the manufactured component is affected as resin starved and resin rich zones become defect sites [6]. In many cases, it is not possible to observe visually the flow of the resin as the materials may be non-transparent or have a complex shape, so alternative methods have to be used to monitor the process.

Fibre optic sensors can be embedded readily into composite materials [1, 5], with their impact on the integrity of the structure being dependent on the orientation of the optical fibre with respect to the reinforcing fibres of the composite material [7]. The use of optical fibre sensing technologies to monitor composite material processes of infusion and cure has been reported widely, exploiting devices such as Fresnel refractometers [8, 9], etched [5] and tapered [10] fibres, long period gratings (LPGs) [11], fibre Bragg gratings (FBGs) [12] and tilted fibre Bragg gratings (TFBGs) [11] embedded within the material. Table 1 provides a summary of the capabilities of some common optical and non-optical sensing technologies for composite material processing.

Sensors that are sensitive to the surrounding refractive index (RI) can be used for two purposes - to detect the presence or absence of resin and to monitor the change in RI of the resin during cure, which is correlated with the degree of cure [11, 13]. Other approaches have relied upon the measurement of the development of strain within the composite material, which has been shown to be correlated with the degree of cure, using FBGs fabricated in single mode [12] and in highly birefringent fibres [13].

Etched fibre sensors, where the cladding of the fibre has been partially or completely removed, or tapered optical fibres, where the diameter of the fibre is reduced by heating and stretching to allow the evanescent wave of the propagating mode to interact with the surrounding medium, have been used to allow the detection of the presence of resin at the sensor location $[1,5,14-16]$.

Fresnel refractometers, which rely on the RI dependence of the reflection of light from the cleaved end of an optical fibre, can provide an indication of the presence of resin and are able to monitor the cure of resins even when the RI of the resin exceeds that of the fibre [9].

LPGs and TFBGs are mode coupling devices, where the grating promotes the coupling of light from the core mode to cladding modes. The interaction of the evanescent field of the cladding modes causes the coupling conditions, and thus the 
TABLE I

Capabilities of some optical and non-optical sensing technologies for composite process monitoring

\begin{tabular}{lll}
\hline \hline Type of sensor & Resin flow and/cure sensing & \multicolumn{1}{c}{ Comment } \\
\hline \hline Standard long period grating (LPG) [24]; standard & Step change in attenuation of an LPG (TFBG) spectrum & Only an array of LPGs (TFBGs) \\
tilted fibre Bragg grating (TFBG) [14] & detected resin arrival. & A serial array of LPGs (TFBGs) at different wavelengths \\
& detected resin flow. & flow. This limits the spatial \\
resolution. & Cure was detected by LPG wavelength shift in response to
\end{tabular}

refractive index change of resin.

TFBG detected cure via the change in spectral attenuation due to refractive index change of resin.

Standard fibre Bragg grating (FBG) [22]

Tapered (or etched) fibre section [22]
Arrival of resin detected by step shift of FBG wavelength as

the resin and mould were at different temperatures.

A serial array of FBGs at different wavelengths detected resin flow.

Arrival of resin is detected as step change in transmission of

light in tapered/etched fibre section.

A serial array of tapers or etched sections detected resin

flow.

Change in attenuation of transmitted light, caused by

refractive index change of resin, was used as a measure of cure.

Fluorescence monitoring:

Two configurations used; either (i) the resin is doped

with fluorescent dye molecules and the fluorescence is monitored by using the evanescent field or the Fresnel reflection from an embedded optical fibre, or (ii) the embedded optical fibre itself is immobilised with the fluorescent dye molecules [24].

\section{Ultrasonic sensors:}

Sensors are non-invasive as sound impulses are transmitted through the mould and laminate without contact to the laminate [26].

High reliability, performance, low cost, and easy integration

Thermocouple sensors [27]:

Micro-thermocouples embedded into preform gave indirect measurements.

Dielectric sensor array [28]:

Sensor requires direct contact or is embedded into preform. Electric field applied to the bottom aluminium plate was transmitted through the laminate to the top polycarbonate plate of the mould to which the sensor array was attached.

Pressure sensors:

Piezo-resistive pressure sensors were embedded,

demonstrating they can be used in conductive carbon fibre composite [29].

\section{Carbon fibre sensor:}

Consists of embedded pair of parallel carbon fibres (typically $3 \mathrm{~mm}$ apart and $50 \mathrm{~cm}$ long) forming a parallel plate capacitor when connected to a voltage supply [30].

An electrolyte is created by adding Lithium ions to the

The measured fluorescence intensity is proportional to the length of optical fibre sensor covered in resin and sensor length greater than $1 \mathrm{~m}$ was used [25].

Wavelength shift of the fluorescence maximum or the intensity of the fluorescence [24] is used to detect cure; including reaction kinetics, glass transition, gelation, and physical aging of cured resin. Responsivity is sensitive to coating thickness.

Monitors resin arrival (flow front position) and velocity [26].

Monitors cure, gelation, vitrification and laminate thickness

[26].

Detected acoustic signals depend on the fibre volume content.

Detects resin arrival through temperature measurement [27] when resin and mould are at different temperatures. Performance/sensitivity depends on this temperature difference.

Exploits the change in capacitance and conductance to detect flow-front location continuously.

Cure is monitored by spectroscopic measurements of the change in dielectric properties of resin at multiple locations simultaneously without embedding the sensor array. Also measured is temperature and viscosity simultaneously.

Measures the vacuum hold, resin flow and pressure buildup.

Only an array of FBGs can give
direction of flow.
Cure is monitored by the FBG via
strain measurement.
Only an array of the sensors can
give direction of flow. This limits
the spatial resolution.

Only an array of FBGs can give direction of flow.

Cure is monitored by the $\mathrm{FBG}$ via

Only an array of the sensors can the spatial resolution.

Cannot detect flow direction.

Dissolving of fluorescent

molecules into resin is extra

processing step that affects resin properties.

Sensor not ideal when in contact with preform fibre.

Accurate detection of flow direction is possible for small mould thicknesses.

Each measurement requires a receiver-transmitter pair probes.

Sensor array is needed for detecting flow direction. The sensor is intrusive.

An array of sensors needed to detect flow direction. The need to generate electric field limits the choice of the mould material.

Cannot detect flow direction. Temperature fluctuation reduces sensitivity.

Sensor is intrusive $(12 \times 4 \mathrm{~mm}$ in size).

Detects resin flow continuously when flow is parallel, and detects step change when flow is perpendicular to the length of the sensor.

The phase between the input voltage and voltage across the fibre capacitor was used as a measure of resin flow [30].
Cannot detect flow direction.

Ion seeding can influence resin viscosity, also the process to produce the carbon fibre sensor is complex.

resin. The flow of resin between the two fibres therefore influences the electrical field.

transmission spectrum of the device, to be sensitive to changes in the RI of the surrounding material. Both devices can indicate the arrival of the resin at the sensor location, and can be used to subsequently monitor the cure of the resin $[11,14]$.

To monitor resin flow, in general there is a requirement to include a number of discrete sensors and map the arrival time of the resin at each sensor. The use of an LPG as a liquid level sensor has been reported, enabling the proportion of the device covered by a liquid to be determined [17], which can be used to monitor the flow of resin along the device. However, the configuration reported did not provide information on the direction of the flow over the sensor.

The determination of the direction of flow of the resin is particularly important in complex shaped preforms [18], as it enables the appropriate resin inlet gates to be regulated in order to control the amount of resin pumped into the preform. 
The concept of a directional flow sensor was introduced by Buggy et al. [19] where they immersed a chirped long period grating in Cargille RI oil causing a change in the shape of the resonance band of the LPG's transmission spectrum. The shape of the band was dependent on whether the shorter periods or longer periods of the LPG were covered. This approach was also shown to have the potential to work with epoxy resin systems [20]. This work is extended here to provide a detailed study and comparison of the use of continuously-chirped and step-chirped LPGs to determine the direction of flow of a UV-cured epoxy resin. The sensors are further used to monitor the cure of the resin which is correlated with measurements from a fibre optic Fresnel refractometer.

A range of optical techniques have been investigated for composite material processing, with demonstrations of the ability to detect the presence of resin using a single sensor [20, $21]$, to measure resin flow during the infusion when the sensors are spatially multiplexed [14, 22], and to monitor the cure of the resin $[11,21,22]$. Superimposed fibre grating sensors, offering strain-temperature discrimination, have also been demonstrated for cure monitoring [23]. The detection of the direction of resin flow using these techniques is possible only with spatially multiplexed sensors, and the spatial resolution depends on sensor length and sensor spacing. In this paper we demonstrate that it is possible to monitor the presence of resin, resin flow and its direction, and cure using a single CCLPG or SCLPG sensor. While the performance of the CCLPG or SCLPG as resin flow and cure sensors is comparable to the performance of other optical sensors, the flow direction monitoring capability by a single CCLPG or SCLPG offers the capability to detect flow and flow direction with improved spatial resolution. Additionally, the multiplexing of a number of CCLPGs or SCLPGs will provide monitoring capabilities over an extended area of the composite preform. Table 1 provides a summary of the capabilities of some common optical and non-optical sensing technologies for composite material processing.

TABLE 1: Capabilities of some optical and non-optical sensing technologies for composite process monitoring

\section{A. Long Period Gratings}

LPGs have periods which range typically from $100 \mu \mathrm{m}$ to 1 $\mathrm{mm}[31,32]$ and promote coupling between the propagating core mode and one or more of the co-propagating cladding modes. This results in a transmission spectrum that consists of a number of resonance bands centred at wavelengths determined by the phase matching condition [33] described by (1):

$$
\lambda=\left[n_{\text {eff }}(\lambda)-n_{\text {clad }}^{i}(\lambda)\right] \Lambda
$$

Where $\lambda$ is the coupling wavelength, $n_{\text {eff }}$ is the effective refractive index of the propagating core mode, $n_{\text {clad }}^{i}$ is the effective refractive index of the $i^{\text {th }}$ cladding mode and $\Lambda$ is the period of the LPG. The attenuation in the resonance bands increases when the length of the LPG is increased. While it has been shown that it is possible to create an LPG of length equivalent to 2 periods using the electric arc approach, in practice, the typical length of an LPG is of the order of 10's of $\mathrm{mm}$. Composite materials that are processed in laboratory environments range from 10's of $\mathrm{mm}$ to several metres in length, while industrial components can be of the order of 10's of metres long, which make the composites suitable for monitoring using LPG sensors. The transmission spectrum of an LPG covers a relatively large wavelength bandwidth which can use up most of the bandwidth available from the light source used for the interrogation. To use a number of LPG sensors, multiplexing schemes ranging from time division [34] to optical frequency domain reflectometry [35] have been employed.

The LPG's sensitivity to changes in the RI of the surrounding medium derives from the phase matching condition's dependence on the effective refractive index of the fibre's cladding modes. The effective refractive index of the cladding mode, which is determined by the difference between the RI of the cladding and that of the surrounding environment, changes along with surrounding RI. This leads to a shift in the wavelength of the resonance bands.

When an LPG has a period that varies from one end of the grating to the other, for example linearly, it is referred to as a chirped long period grating [36]. The resonance bands of a chirped LPG generally have a broader bandwidth than those with a uniform period. Figure 1a illustrates the structure of a linearly chirped LPG where the period increases continuously from one end of the grating to the other.

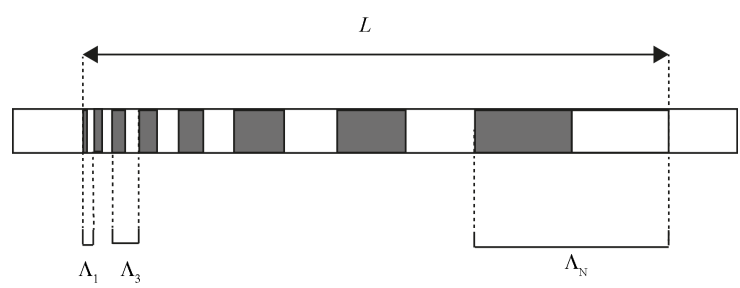

(a)

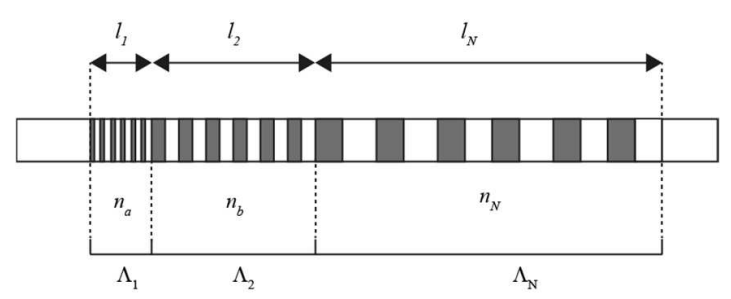

(b)

Fig. 1. (a) represents a continuously chirped long period grating (CCLPG) where $\Lambda_{i}$ is the period and $L$ is the length of the LPG. (b) Represents a step chirped long period grating (SCLPG) where $n_{i}$ is the number of periods in each section, $l_{i}$ is the length of each section. The grey areas represent sections of fibre that have been exposed to UV irradiation.

A step chirped long period grating (SCLPG) is one in which the periods are varied in large discrete steps and thus the LPG 
consists of a number of sections where the period in each section is constant (Fig. 1b).

\section{EXPERIMENT}

A CCLPG, with an effective length of $35 \mathrm{~mm}$ and periodicity varying linearly over its length, from $400 \mu \mathrm{m}$ to $420 \mu \mathrm{m}$, was fabricated in a single mode fibre with a cut off wavelength of $627 \mathrm{~nm}$ (Fibercore PS750) using a UV laser (266nm) and the point-by-point inscription method [37]. The transmission spectrum of the LPG was monitored by coupling light from a tungsten-halogen light source to a high resolution CCD spectrometer (Ocean Optics HR4000). This chirp rate was chosen so that the resonance bands in the transmission spectrum would be broadened but not spectrally overlap. The length of the LPG was chosen as it provided a transmission spectrum with resonance bands of sufficient extinction for this experiment while allowing the LPG to be handled easily.

Figure 2 shows the normalised transmission spectrum of a CCLPG with a period range of $400 \mu \mathrm{m}-420 \mu \mathrm{m}$, along with those of uniform-period LPGs with periods $400 \mu \mathrm{m}$ and 420 $\mu \mathrm{m}$. The extreme wavelength bounds of the CCLPG bands are coincident with the wavelengths of the resonance bands of the two uniform period LPGs, as expected.

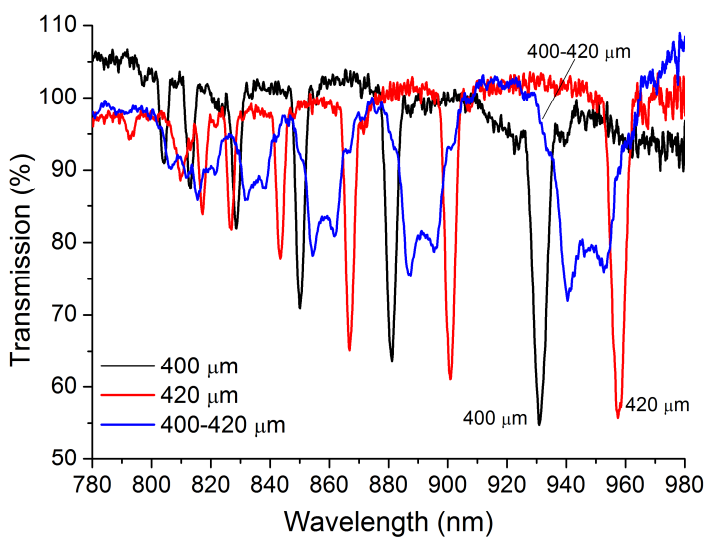

Fig. 2. Transmission spectra of uniform period LPGs with periods of $400 \mu \mathrm{m}$ and $420 \mu \mathrm{m}$ and a CCLPG with a period range of $400 \mu \mathrm{m}-420$ $\mu \mathrm{m}$. All LPGs had a length of $35 \mathrm{~mm}$ and were written in a fibre with a cut off wavelength of $627 \mathrm{~nm}$ (Fibrecore PS750).

Figure 2 also shows that, while the individual resonance bands of the uniform period LPGs have a relatively narrow bandwidth, the bands of the CCLPG exhibit an increased bandwidth and a decrease in the resonance depth. This is because the wavelength coupled to the cladding mode varies as a function of the position along the CCLPG.

An SCLPG with the same parameters was also fabricated, with its transmission spectrum shown in Fig. 3. However, instead of a continuous chirp, there was a discrete step change in period of $2 \mu \mathrm{m}$ from one group of periods to the next. Again, the width of each band is bound by the upper and lower periods that constitute the chirped LPG. Each of the resonance bands of the SCLPG is split into two distinct bands because of the phase shift that is introduced by the discontinuous chirp (i.e. step chirp). The SCLPG spectrum thus resembles the spectrum of a phase-shifted long period fibre grating [38].

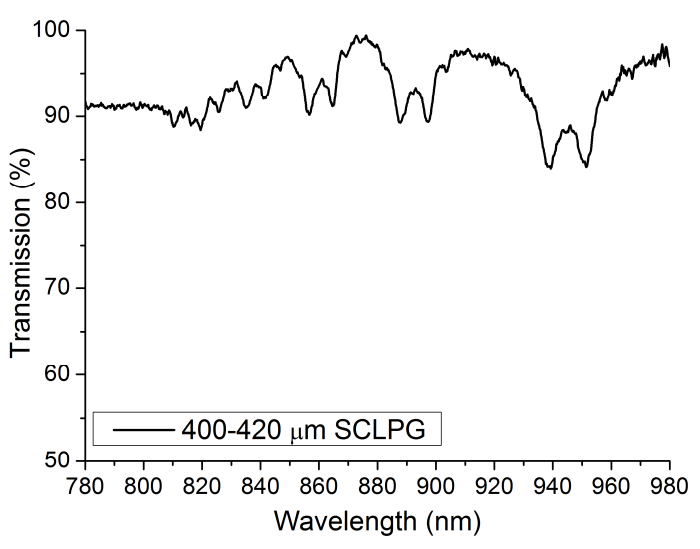

Fig. 3. Transmission spectrum of a step chirped LPG of length $35 \mathrm{~mm}$ with a period ranging from $400 \mu \mathrm{m}$ to $420 \mu \mathrm{m}$ and a step chirp of $2 \mu \mathrm{m}$. The LPG was written in a fibre with a cut off wavelength of $627 \mathrm{~nm}$ (Fibrecore PS750).

\section{A. Epoxy Resin Flow}

The directional flow measurement capabilities of the chirped LPGs were assessed using a UV cured epoxy resin which had an uncured RI of 1.4230 , specified at $589 \mathrm{~nm}$, and a viscosity of $50-100 \mathrm{cPs}$ at $100 \mathrm{RPM} / 23{ }^{\circ} \mathrm{C}$ (Epotek OG-134) [39]. The CCLPG was secured along the inside of a long, thin plastic channel. This was positioned at an incline of $1.2 \pm 0.1^{\circ}$ relative to the table surface, to allow the resin to flow slowly over the grating in one direction. $200 \mu \mathrm{l}$ of resin was pipetted into the channel at the highest point (see Fig. 4).

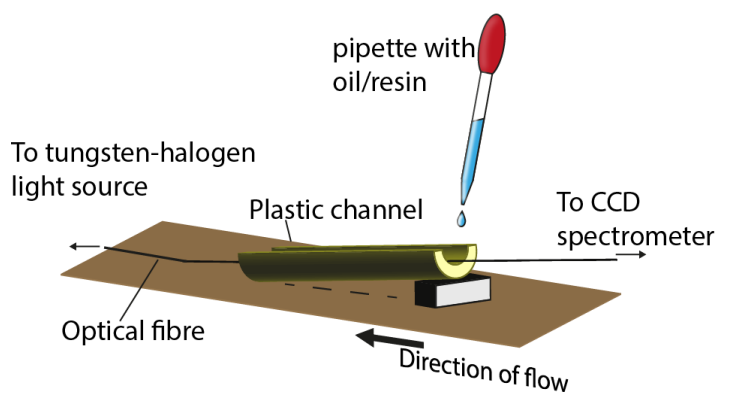

Fig. 4. Experimental configuration for the directional flow experiment The plastic channel was inclined at an angle and the resin was added at the highest point of the channel.

The incline of the channel was then reversed so the resin would flow in the opposite direction relative to the chirp of the grating. In order to identify the location of the front of the resin flow, a small amount of dye was added to the resin. Blue food colouring was pipetted into the resin and the mixture was shaken vigorously (to ensure uniform distribution of the dye), and the flow was recorded with a digital camera to determine the length of the LPG covered by the resin. 


\section{B. Epoxy Resin Curing}

The CCLPG and SCLPG were also used to monitor the RI of the resin while the resin cured. The resin was contained in a small Teflon cell and the CCLPG and SCLPG were placed within the liquid resin. The fibre ends were fixed to fibre holders, using cyanoacrylate adhesive, in order to keep the LPGs taut. To measure the change in RI during cure, optical fibre Fresnel based refractometers [9] were also placed inside the resin to provide independent measurements at the same spatial location of the grating sensors. The Fresnel refractometer provided localised measurements that could be readily calibrated. The RI is determined by calculating the ratio between two light signals reflected from two cleaved optical fibre ends, the sensing probe embedded in resin and a reference probe placed in air.

The property of the LPGs which was exploited for monitoring the curing of the resin is the shift in the central wavelength of a resonance band caused by the associated change in the RI of the resin. The RI dependence of the wavelength shift of the resonance bands of a uniform period LPG were calibrated by immersing the LPG in a series of Cargille oils of known refractive indices. A calibration function was obtained and used later when monitoring the resin cure to convert wavelength shifts of the resonance bands of the CCLPG and SCLPG directly into the RI of the resin under cure. Figure 5 shows the response of the resonance band corresponding to the $\mathrm{LP}_{07}$ mode of a $400 \mu \mathrm{m}$ uniform period LPG to changing surrounding RI obtained using a series of Cargille oils. The RI values were plotted against wavelength shift, so that the function fitted to the curve could be used directly to interpret the RI during measurement experiments, without having to obtain the inverse function. A $7^{\text {th }}$ order polynomial was used to generate a transfer function relating the wavelength shift to the surrounding RI. The transfer function obtained from the calibration curve of Fig. 5 was the same as that obtained from the separate calibrations for the CCLPG and SCLPG, and the transfer function has been used to interpret the RI during the measurement experiments.

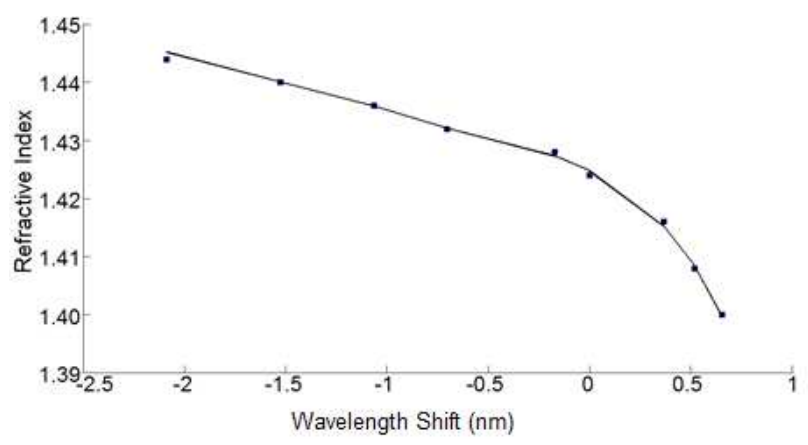

Fig. 5. Response to surrounding RI of the resonance band corresponding to coupling to the $\mathrm{LP}_{07}$ mode of an LPG of uniform period of $400 \mu \mathrm{m}$. The data points were fitted with a $7^{\text {th }}$ order polynomial to generate a function relating the wavelength shift to the surrounding RI.

The experimental configuration used to monitor the cure of the UV-cured resin is shown in Fig. 6. For the Fresnel refractometer, a superluminescent diode (SLD) (Covega 1005) with a bandwidth of $100 \mathrm{~nm}$, centred at $1550 \mathrm{~nm}$, and an output power of $15 \mathrm{~mW}$ was intensity modulated at a frequency of $1 \mathrm{kHz}$ by modulating the injection current. The output was coupled into a network of $3 \mathrm{~dB}$ directional couplers. One arm of coupler 2 was terminated by a cleaved surface and was placed in the resin, allowing the reflection from the resin/fibre interface to be monitored by a photodiode, $\mathrm{pd} 2$. The other output was used to provide a reference by measuring the reflections at the air/fibre interface. This signal was monitored using a second photodiode, pd1. The outputs of the photo detectors (New Focus, Model 2011) were monitored using lock-in amplifiers (National Instruments NI PXI-4462). The RI calibration from the Fresnel refractometer was calculated from the Fresnel expression as described in [11]. An LPG of uniform period $(400 \mu \mathrm{m})$ was also placed in the resin for comparison purposes, along with the CCLPG and SCLPG. A UV lamp, which operated at a central wavelength of $365 \mathrm{~nm}$ (Superlite-UV Lumatec), was placed above the resin and the resin was left to cure for 65 minutes. Data were recorded every 30 seconds during the cure process. The temperature was measured using a thermocouple placed in the resin, close to the LPGs, with readings taken every 30 seconds.

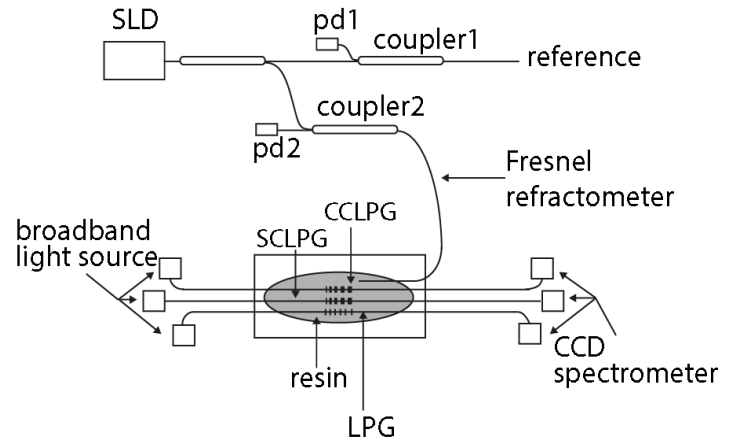

Fig. 6. Experimental configuration for monitoring the cure of a resin. SLD, superluminescent diode laser; CCLPG, continuously chirped long period grating; SCLPG, step chirped long period grating; LPG, long period grating; pdx, photodiode. Adapted from [11].

\section{RESUlTS AND DISCUSSION}

\section{A. Epoxy Resin Flow}

Figure 7 shows the evolution of the resonance band corresponding to the $\mathrm{LP}_{07}$ cladding mode of the CCLPG under the influence of the resin. When the RI of the external surroundings is lower than that of the fibre cladding, total internal reflection (TIR) is the dominant effect responsible for guiding the coupled cladding mode [40]. When an LPG is covered by a material whose RI is lower than that of the fibre cladding, the effective refractive index of the cladding increases, causing a blue wavelength shift of the resonance band and a reduction in the depth of the band [41]. When the resin flow began at the lower period end of the CCLPG, the lower-wavelength side of the resonance band decreased in 
attenuation first, expected as the shorter periods were covered first. For flow in the direction of decreasing period, the converse was true. When the entire length of the CCLPG was covered, the resonance band returned to its original profile/shape with a shift to a lower wavelength corresponding to the change in the surrounding RI caused by the presence of the resin. For flow in the decreasing period direction, the opposite effect was evident.

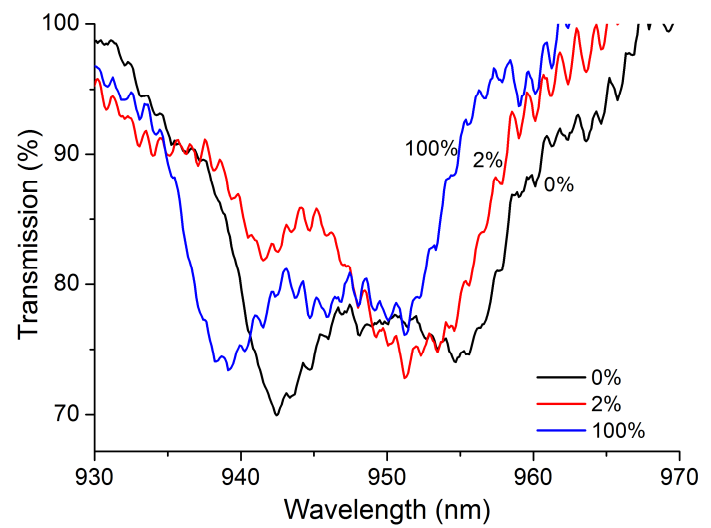

(a)

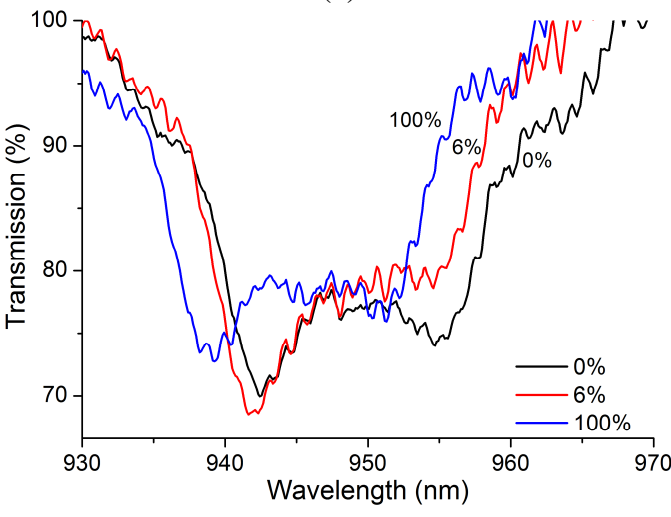

(b)

Fig. 7. The change in the profile of the resonance band corresponding to coupling to the $\mathrm{LP}_{07}$ mode by the CCLPG as the resin flowed in the (a) $400 \mu \mathrm{m}-420 \mu \mathrm{m}$ period direction and (b) $420 \mu \mathrm{m}-400 \mu \mathrm{m}$ period direction. The $\%$ refers to the length of grating covered by the resin during the flow. (a) $2 \%$ and (b) $6 \%$ are the lengths of grating covered where the band profile/shape changes due to flow can be first detected and $100 \%$ corresponds to the entire grating being covered.

It is interesting to note that the onset of the response of the CCLPG resonance band to the resin flow differed depending on the direction of the flow. For flow in the direction of increasing period, a change in the shape of the resonance band was evident after $2 \%$ of the length of the grating was covered for increasing period direction, while for flow in the opposite direction $6 \%$ of the grating length was covered before a change in the band shape could be detected. This is due to the nature of the structure of a CCLPG, in that there are a greater number of periods nearer the $400 \mu \mathrm{m}$ end of the grating than there are at the $420 \mu \mathrm{m}$ side. The effects are more pronounced at the early stages of the flow coverage. At $0 \%$ and $100 \%$ coverage, the profile shape of the resonance band is the same.
The shift of the wavelength corresponds to the change in the RI (the index of the uncured resin is lower than that of the cladding).

To compare the flow measurement capabilities of the SCLPG and CCLPG, the two LPGs were mounted in the same channel. The two LPGs were able to detect the arrival of the resin under near identical conditions. Figure 8 shows the changes in the resonance bands corresponding to coupling to the $\mathrm{LP}_{07}$ mode by the CCLPG and SCLPG over time, respectively, as resin flowed across the length of the gratings. A reference spectrum was recorded with the LPG surrounded by air, and this was subtracted from the "live" spectrum to reveal changes caused by the flow of the resin over the LPG.

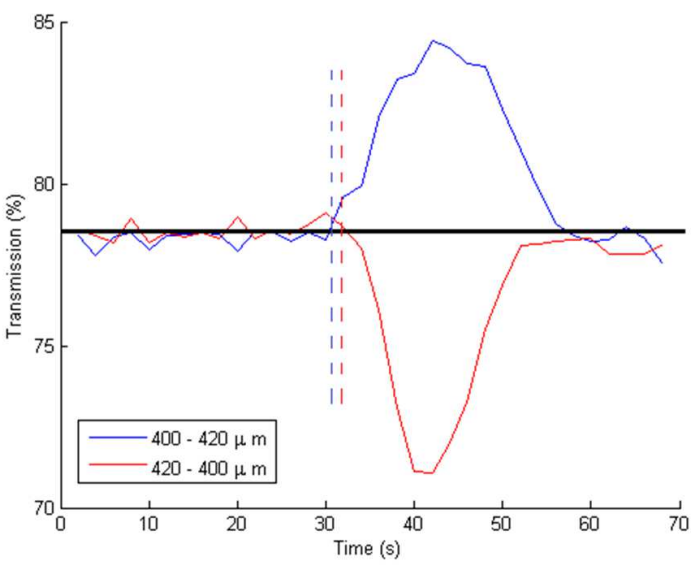

(a)

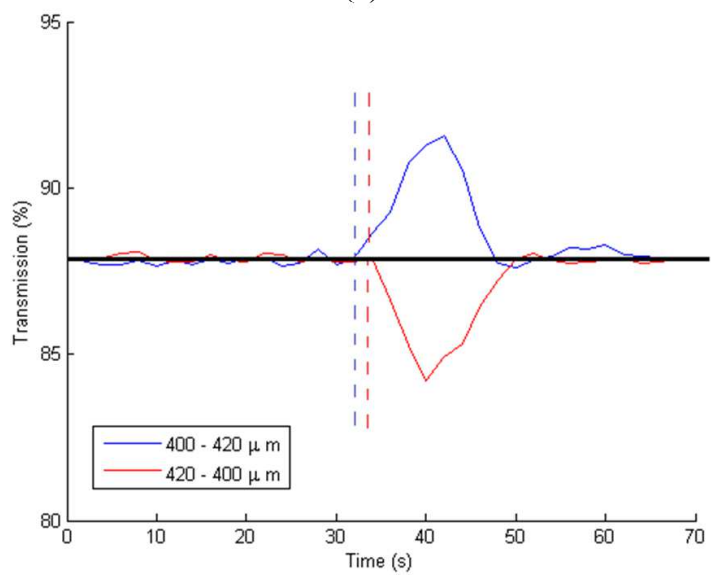

(b)

Fig. 8. Change in the transmission spectrum of (a) the CCLPG and (b) the SCLPG, measured at $940 \mathrm{~nm}$ as resin flowed over the length of the grating in the increasing and decreasing chirp period directions. The blue peak represents resin flow in the $400 \mu \mathrm{m}-420 \mu \mathrm{m}$ period direction and the red trough represents flow in the $420 \mu \mathrm{m}-400 \mu \mathrm{m}$ period direction. The horizontal and vertical lines provide a guide to indicate the onset of change in the shape of the resonance band with flow. $\Lambda$ indicates the grating period.

As can be seen, depending on the direction of the resin flow (increasing or decreasing chirp period direction), the two curves displace in opposing directions. The y axes in Fig. 8a and $8 \mathrm{~b}$ have been scaled the same in order to show the larger changes shown by the CCLPG. The figures also confirm that, 
in the increasing chirp period direction, the resonance band reacts sooner to the introduction of resin than it does in the decreasing chirp period direction, as indicated by the vertical dotted lines (though this is less apparent for the decreasing period, as the resin has to cover a larger distance before sufficient periods of similar length can impact on the transmission spectrum). In the case of flow from the shorter period direction, a number of periods are covered in a shorter physical distance and thus a change in the spectrum is seen earlier. This also explains the time lapse between the onset of resin flow and change in spatial profile experienced in the 420 $\mu \mathrm{m}-400 \mu \mathrm{m}$ period direction, as the resin has to cover a relatively longer length of the LPG. Resin was introduced into the flow cell such that it took approximately $30 \mathrm{~s}$ before arriving at the LPG location, allowing the resin to cover the LPG length at a constant speed.

The change in the transmission spectrum exhibited by the SCLPG follows broadly the same trend as that of the CCLPG. However, as it lacks the intermediate periods present in the CCLPG, which can be considered to be equivalent to a loss of resolution, there are differences in response. For flows in the direction of decreasing period, the response of the SCLPG occurs later than that of the CCLPG. In addition, the transmission spectrum of the SCLPG stops responding to resin flow sooner than the CCLPG. Although the resin completely covered the two gratings after approximately $23 \mathrm{~s}$, only the spectral profile of the CCLPG changed for the entire duration of the flow along the grating, with the changes in the SCLPG ending earlier.

\section{B. Epoxy Resin Curing}

The thermal state of the UV-cured resin was characterised during the cure process, as shown in Fig. 9, before the LPG and Fresnel sensors were deployed in further experiments to monitor the resin cure. The thermocouple showed that there was a $2{ }^{\circ} \mathrm{C}$ decrease in the temperature of the resin, following an initial rise of about $0.5^{\circ} \mathrm{C}$.

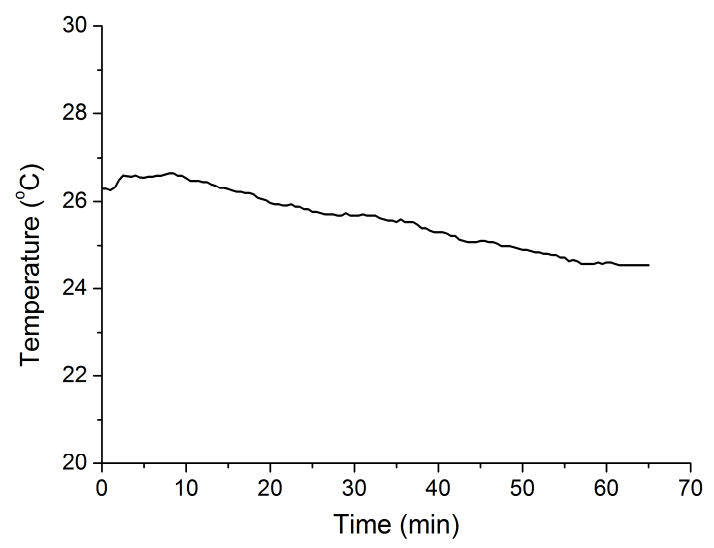

Fig. 9. Temperature of the epoxy resin as measured by a thermocouple during the cure process.

This rise in temperature can be attributed to an exothermic reaction that occurred shortly after the UV lamp was turned on. The thermal characterisation experiments showed a maximum temperature excursion of the order of $0.6{ }^{\circ} \mathrm{C}$ recorded by a thermocouple embedded in the resin during the cure process. Such a temperature change will contribute a maximum error in the RI measurement of $5.76 \times 10^{-6}$ caused by the thermo-optic coefficient of the optical fibre $\left(9.6 \times 10^{-}\right.$ ${ }^{6} /{ }^{\circ} \mathrm{C}$ [42]), which is relatively insignificant. An additional error, also expected to be insignificant, in the measured RI may also be introduced by the RI change of the resin caused by the temperature excursion. This error could not be calculated as the thermo-optic coefficient of the UV-cured resin was unknown. As an example, the thermo-optic coefficient of RTM6, a thermosetting resin, is approximately $3.58 \times 10^{-4} /{ }^{\circ} \mathrm{C}$ [43].

Figures 10 show the wavelength shifts of the resonance band corresponding to the $\mathrm{LP}_{07}$ modes of the CCLPG, SCLPG and a uniform period LPG as the resin was being cured. As the bandwidth of the attenuation band is relatively broad for its wavelength to be accurately located, the central wavelengths of the chirped LPGs were determined by fitting a polynomial to the spectral feature and differentiating, using a routine written in LabVIEW. The shifts in the measured central wavelengths of the resonance bands after the cure cycle was complete, corresponding to the change in RI of the resin, were comparable for the CCLPG and SCLPG, at $-1.1 \pm 0.1 \mathrm{~nm}$ and $-1.1 \pm 0.1 \mathrm{~nm}$ respectively. The resonance band of the uniform period LPG shifted by $-0.6 \pm 0.1 \mathrm{~nm}$. This shift may be due to the LPG being spatially displaced from the other two gratings, so that it was located in a region of the resin that did not get exposed to the same UV flux and thus did not experience the same degree of cure. This was confirmed by a second Fresnel refractometer placed at a similar spatial position. It also showed a diminished response suggesting that the degree of cure was lower.

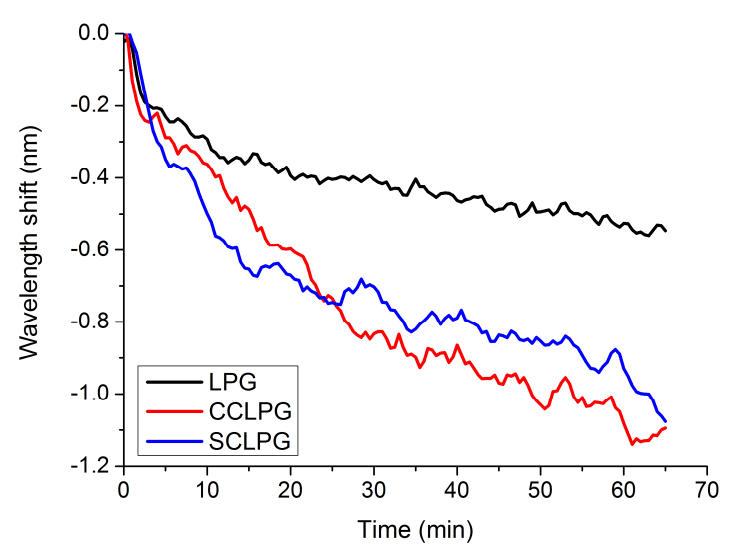

Fig. 10. Wavelength shift of the resonance band corresponding coupling to the $\mathrm{LP}_{07}$ mode by the CCLPG and SCLPG with period ranging from $400 \mu \mathrm{m}-420 \mu \mathrm{m}$, and by the $400 \mu \mathrm{m}$ uniform period LPG measured during the curing of the epoxy resin over 65 minutes.

The measured wavelength shifts were converted to equivalent changes in RI with the aid of the calibration curves 
of Fig. 5. The responses of the fibre gratings to the changes in the RI were compared with the changes in RI measured by the Fresnel refractometer during the same experiment (Fig. 11 13). The correlation coefficient being used is the adjusted $R^{2}$, which not only takes into account how well the data points fit the trendline, but also the number of predictors that are involved (" $x$ " variables). There was a good linear correlation of 0.96 between the wavelength shifts of the CCLPG and the change in RI measured with the Fresnel refractometer. The linear correlations obtained for the SCLPG and LPG were 0.91 and 0.97 , respectively.

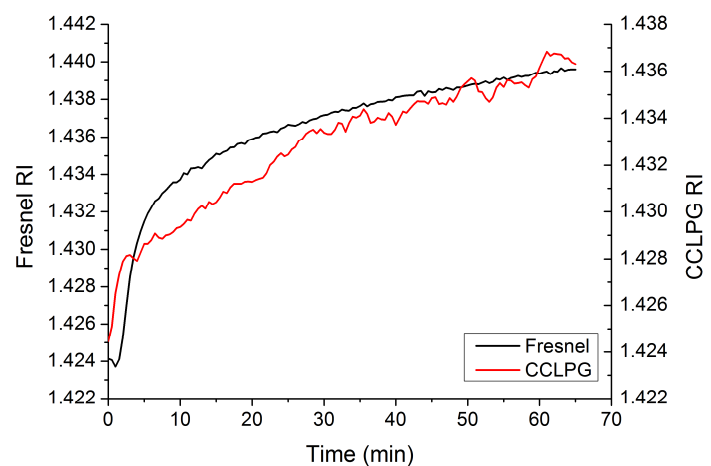

(a)

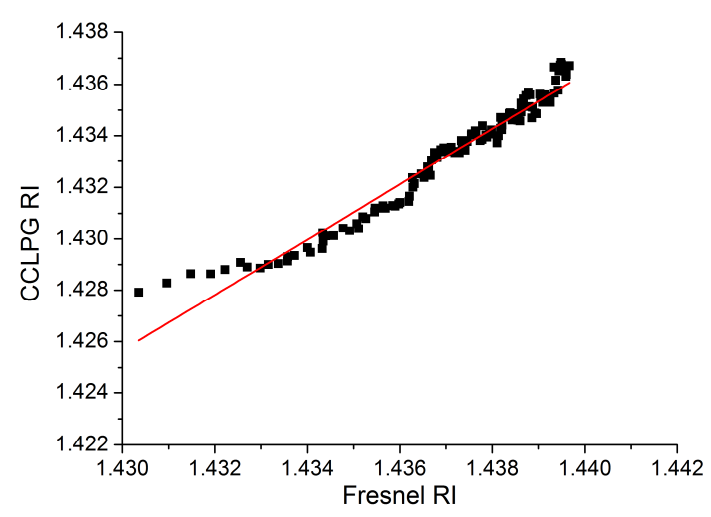

(b)

Fig. 11. Correlation of RI changes a measured by the Fresnel refractometer and by a CCLPG with a period range of $400 \mu \mathrm{m}-420 \mu \mathrm{m}$, obtained during epoxy resin cure. (a) Shows the changes in RI measured by the CCLPG (red curve) and Fresnel refractometer (black curve) and (b) shows the plot of CCLPG-measured RI against the RI measured using a Fresnel refractometer. A linear fit (solid line) was added to show a correlation coefficient of 0.96 .

The LPG had a slightly higher correlation due to the narrower bandwidth of the resonance bands, which allowed the central wavelength to be determined with higher resolution. A linear correlation was expected as, even though the Fresnel and the LPG sensors operated at different wavelengths and thus through dispersion the refractive indices the resin experienced were different, the same trend would be observed. These measurements indicate that sensitivity to cure-induced refractive indices was not greatly compromised when using the broader resonance bands of the CCLPG and SCLPG, while the use of these chirped LPG structures also allowed the determination of the direction of resin flow.

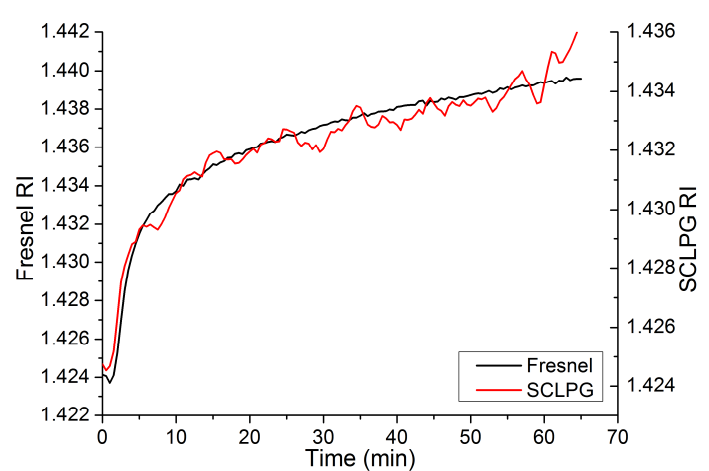

(a)

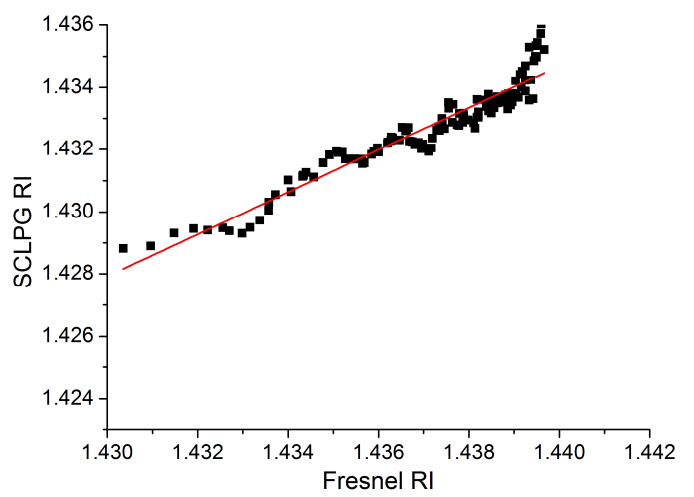

(b)

Fig. 12. Correlation of the RI changes measured by the Fresnel refractometer and by a SCLPG with a period range of $400 \mu \mathrm{m}$ to $420 \mu \mathrm{m}$, obtained during epoxy resin cure. (a) Shows the changes in RI measured by the SCLPG (red curve) and Fresnel refractometer (black curve) and (b) shows the plot of SCLPG-measured RI against the RI measured using the Fresnel refractometer. A linear fit (solid line) was added to show a correlation coefficient of 0.91 . 


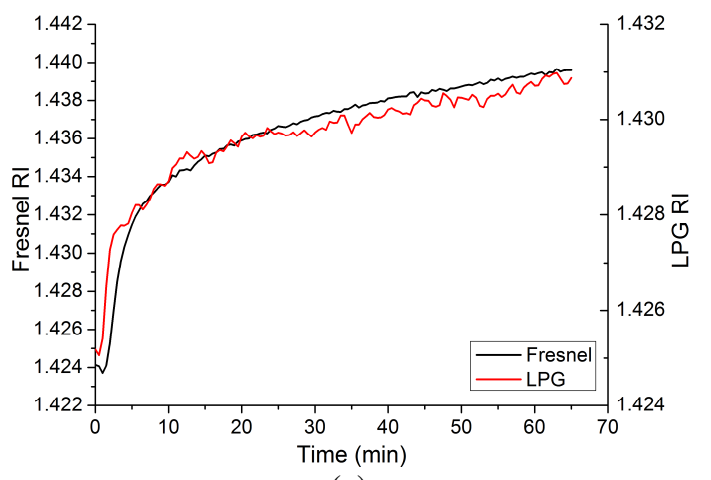

(a)

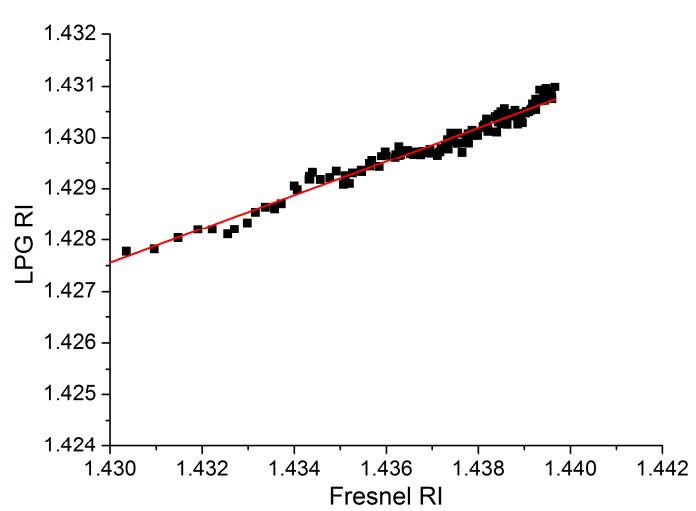

(b)

Fig. 13. Correlation of RI changes measured by the Fresnel refractometer and by an LPG with a period of $400 \mu \mathrm{m}$ during epoxy resin cure. (a) Shows the changes in RI measured by the LPG (red curve) and by the Fresnel refractometer (black curve) and (b) shows the LPG-measured RI plotted against the RI measured using a Fresnel refractometer. A linear fit (solid line) was added to show a correlation coefficient of 0.97 .

\section{SUMMARY AND CONCLUSIONS}

A CCLPG was used as a directional flow sensor and as a resin cure sensor. The change in the shape of the resonance band was shown to be dependent on the direction of flow of the resin with respect to the grating chirp orientation. When the resin flowed in the increasing chirp direction, the resonance band appeared to move toward longer wavelengths. In the decreasing chirp direction the resonance band moved to the left (shorter wavelengths), with the resonance band having more prominence on the blue edge of its spectrum. The sensitivities of a CCLPG and a SCLPG, both with a total chirp of $20 \mu \mathrm{m}$, were compared. For directional flow measurement, the CCLPG was shown to be more responsive than the SCLPG. This implies that the CCLPG would make a more sensitive directional flow sensor when compared to a SCLPG. The detection provided (transmission spectrum profile change) by the SCLPG followed the same trend as that of the CCLPG but it lacked the intermediate periods provided by the CCLPG, leading to a reduction in resolution. The CCLPG can also be used for monitoring flow of materials with both higher and lower refractive indices than that of the fibre cladding.

The CCLPG and SCLPG were used to monitor the cure of the resin and the responses of the resonance bands were compared with that of a uniform period LPG. The experiment demonstrated that the sensitivity was not affected when using a CCLPG or SCLPG during the cure process, despite the increased width of the resonance bands. The change in RI during cure also correlated well with the results from the Fresnel refractometer, with correlation coefficients of 0.96 for the CCLPG and 0.91 for the SCLPG. Though the uniform period LPG still had the higher correlation of 0.97, it cannot be used to detect directional flow. The CCLPG is able to perform both directional flow sensing and cure monitoring using a single sensor. While the broad spectral width of the resonance bands compromises the accurate measurement of the central wavelengths of the band, this could be mitigated by reducing the magnitude of the chirp and at the same time using a shorter period or by increasing the number of grating planes written while keeping the overall length of the grating fixed.

This experiment involved resin flowing along the axis of the fibre from one end to the other. In real world situations this may not be the case (e.g. resin may reach the centre of the CCLPG first or reach different parts of the sensor at the same time). However, due to the nature of the CCLPG having a linearly varying period, it may be possible to determine the location of the resin along the LPG.

\section{ACKNOWLEDGMENT}

The authors acknowledge the support of the Engineering and Physical Sciences Research Council (EPSRC) UK, via grant $\mathrm{EP} / \mathrm{H} 02252 \mathrm{X} / 1$. The underlying data can be found at http://dx.doi.org/10.17862/cranfield.rd.4223643.

\section{REFERENCES}

[1] C. Keulen, B. Rocha, M. Yildiz, and A. Suleman, "Embedded fiber optic sensors for monitoring processing, quality and structural health of resin transfer molded components," J. of Phys.: Conf. Series, vol. 305, no. 1, pp. 1-9, Jul. 2011.

[2] C-C. Chiang, "Curing Monitoring of Composite Material Using Embedded Fiber Bragg Grating Sensors," in Advances in Composite Materials - Analysis of Natural and Man-Made Materials, $\mathrm{P}$ Tesinova, Ed., INTECH, Croatia, 2011, pp. 345-360.

[3] J. Galy, A. Sabra, and J-P. Pascault, "Characterization of epoxy thermosetting systems by differential scanning calorimetry," Polymer Eng. Sci., vol. 26, no. 21, pp. 1514-1523, Nov. 1986.

[4] A. A. Skordos, P. I. Karkanas, and I. K. Partridge, "A dielectric sensor for measuring flow in resin transfer moulding," Meas. Sci. Technol., vol. 11 , pp. 25-31, Oct. 2000.

[5] C. Keulen, M. Yildiz, and A. Suleman, "Multiplexed FBG and etched fiber sensors for process and health monitoring of 2-\&-3-D RTM components," J Rein Plast Comp, vol. 30, no. 12, pp. 1055-1064, Aug. 2011 .

[6] M. Bannister, A. Baker, A. Garg, A. A. Kharibi, and Y. W. Mai, "Mechanical property measurement" in Composite materials for aircraft structures, 2nd ed., A. Baker, S. Dutton, and D. Kelly, Eds., AIAA, 2004.

[7] S. S. J. Roberts. and R. Davidson, "Mechanical properties of composite materials containing embedded fibre optic sensors," in Proc. SPIE, vol. 1588, 2001, pp. 326-341.

[8] C. B. Kim, and C. B. Su, "Measurement of the refractive index of liquids at 1.3 and 1.5 micron using a fibre optic Fresnel ratio meter," Meas. Sci. Technol., vol. 15, pp. 1683-1686, Jul. 2004.

[9] A. Dimopoulos, S. J. Buggy, A. A. Skordos, S. W. James, R. P. Tatam, and I. K. Partridge, "Monitoring cure in epoxies containing carbon nanotubes with an optical-fiber Fresnel refractometer," J. Appl. Polym. Sci., vol. 113, no. 2, pp. 730-735, Mar. 2009.

[10] R. Jarzebinska, E. Chehura, S. W. James, and R. P. Tatam, "Multiplexing a serial array of tapered optical fibre sensors using 
coherent optical frequency domain reflectometry," Meas. Sci. Technol. Vol. 23, pp. 105203 (8pp), Aug. 2012.

[11] S. J. Buggy, E. Chehura, S. W. James, and R. P. Tatam, "Optical fibre grating refractometers for resin cure monitoring," J. Opt. A: Pure and Appl. Opt., vol. 9, pp. S60-S71, May 2007.

[12] M. J. O’Dwyer, G. M. Maistros, S. W. James, R. P. Tatam, and I. K. Partridge, "Relating the state of cure to the real-time internal strain development in a curing composite using in-fibre Bragg gratings and dielectric sensors," Meas. Sci. Technol., vol. 9, no. 8, pp. 1153-1158, Apr. 1998.

[13] E. Chehura, A. A. Skordos, C-C. Ye, S. W. James, I. K. Partridge, and R. P. Tatam, "Strain development in curing epoxy resin and glass fibre/epoxy composites monitored by fibre Bragg grating sensors in birefringent optical fibre," Smart Mater. Struct., vol. 14, no. 2, pp. 354362, Feb. 2005.

[14] E. Chehura, R. Jarzebinka, E. F. Reia Da Costa, A. A. Skordos, S. W. James, I. K. Partridge, and R. P. Tatam, "Multiplexed fibre optic sensors for monitoring resin infusion, flow, and cure in composite material processing," in Proc. SPIE, vol. 8693, 2013, pp. F1-F7.

[15] N. Gupta, and R. Sundaram, "Fiber optic sensors for monitoring flow in vacuum enhanced resin infusion technology (VERITy) process," Compos. A, vol. 40, no. 8, pp. 1065-1070, Aug. 2009.

[16] S. H. Ahn, W. I. Lee, and G. S. Springer, "Measurement of the threedimensional permeability of fiber preforms using embedded fiber optic sensors," J. Compos. Mater., vol. 29, no. 6, pp. 714-733, Apr. 1995.

[17] S. Khaliq, S. W. James, and R. P. Tatam, "Fibre optic liquid level sensor using long period gratings," Opt. Lett., vol. 26, no. 16, pp. 1224-1226, Aug. 2001.

[18] R. Di Sante, "Fibre optic sensors for structural health monitoring of aircraft composite structures: recent advances and applications," Sensors, vol. 15, no. 8, pp. 18666-18713, Jul. 2015

[19] S. J. Buggy, S. W. James, and R. P. Tatam, "A long period grating based directional flow sensor," in Proc. SPIE, vol. 7004, 2008, pp. 70045P $(4 \mathrm{pp})$.

[20] R. Y. N. Wong, E. Chehura, S. W. James, and R. P. Tatam, "A chirped long period grating sensor for monitoring flow direction and cure of a resin," in Proc. SPIE, vol. 8693, 2013, pp. 86930E.

[21] S. Konstantopoulos, E. Fauster, and R. Schledjewski, "Monitoring the production of FRP composites: A review of in-line sensing methods", Express Polym. Lett., vol. 8, no. 11, pp. 823-840, 2014.

[22] M. Yildiz, N. G. Ozdemir, G. Bektas, C. J. Keulen, T. Boz, E. F. Sengun, C. Ozturk, Y. Z. Menceloglu, and A. Suleman, "An Experimental Study on the Process Monitoring of Resin Transfer Molded Composite Structures Using Fiber Optic Sensors", Journal of Manufactur. Sci. Eng., vol. 134, no. 4, pp. 044502 (6 pages), June 27, 2012.

[23] E. Marin, L. Robert, S. Triollet, and Y. Ouerdane, "Liquid Resin Infusion process monitoring with superimposed Fibre Bragg Grating sensor", Polym. Testing, vol. 31, pp. 1045-1052, 2012.

[24] J. P. Dunkers, J. L. Lenhart, S. R. Kueh, J. H. van Zanten, S. G. Advani, and R. S. Parnas, "Fiber optic flow and cure sensing for liquid composite molding", Opt. Laser. Eng., vol 35, 2001, pp. 91-104.

[25] S. R.M. Kueh, S. G. Advani, and R. S. Parnas, "A Minimalist Sensor System for Mold Filling", in Proc. ICCM-12: smart mater. and smart manufactur., Paper no 179, 2012.

[26] N. Liebers, D. Bertling, M. Kleineberg, and M. Wiedemann, "Monitoring and forecast of resin infusion processes without contact to part", International Symposium on Composites for High Perfomance Applications, 24.-25. Aug. 2016, Braunschweig, Germany.

[27] P. Wang, J. Molimard, S. Drapier, A. Vautrin, and J.C. Minni, "Monitoring the resin infusion manufacturing process under industrial environment using distributed sensors", J. Compos. Mater., vol. 46, no 6, pp. 691-706, 2011.

[28] M. C. Hegg, A. Ogale, A. Mescher, A. V. Mamishev, and B. Minaie, "Remote Monitoring of Resin Transfer Molding Processes by Distributed Dielectric Sensors", J. Compos. Mater., vol. 39, no. 17, pp 1519-1539, 2005

[29] M. K. Moghaddam, M. Salas, I. Ersoz, I. Michels, and W. Lang, "Study of resin flow in carbon fiber reinforced polymer composites by means of pressure sensors", J. Compos. Mater., vol. 0, no. 0, pp. 1-10, 2017.

[30] M. Bakhshi, and A. Horoschenkoff, "Use of carbon fiber sensors to determine the resin flow", in Proc. ICCM-19, pp. 7606-7613, 2013.

[31] D. B. Stegall, and T. Erdogan, "Leaky cladding mode propagation in long-period fiber grating devices," IEEE Photon. Technol. Lett., vol. 11, no. 3, pp. 343-345, Mar. 1999.
[32] S. W. James, and R. P. Tatam, "Optical Fibre Long Period Grating Sensors: Characteristics and Application," Meas. Sci. Technol., vol. 14, no. 5, pp. R49-R61, Mar. 2003.

[33] V. Bhatia, and A. M. Vengsarkar, "Optical fiber long-period grating sensors," Opt. Lett., vol. 21, no. 9, pp. 692-694, May 1996.

[34] H. S. Lin, M. R. Mokhtar, F. A. Rahman, H. A. A. Rashid, M. Roslina, and N. A. M. Arif, "Simultaneous spectral recovery of long-period grating sensor array using optical time-division multiplexing", Optik, vol. 123 , pp. 650-652, 2012.

[35] M. Jiang, D. Chen, and S. He, "Multiplexing Scheme of Long-Period Grating Sensors Based on a Modified Optical Frequency Domain Reflectometry", IEEE Photon Technol. Lett., vol 20, no. 23, pp. 19621964, Dec. 2008.

[36] Z. Wu, X. Dong, and Z. Xu, "Chirp characteristics of long-period fiber gratings," in Proc. SPIE, vol. 4595, 2001, pp. 89-93.

[37] R. Y. N. Wong, E. Chehura, S. E. Staines, S. W. James, and R. P. Tatam, "Fabrication of fiber optic long period gratings operating at the phase matching turning point using an ultraviolet laser," Appl. Opt., vol. 53, no. 21, pp. 4669-4674, Jul. 2014.

[38] H. Ke, K. S. Chiang, and J. H. Peng, "Analysis of Phase-Shifted LongPeriod Fiber Gratings," IEEE Photon. Technol. Lett., vol. 10, no. 11, pp. 1596-1598, Nov. 1998.

[39] EPO-TEK ® OG134 technical data sheet, Epoxy Technology Inc.

[40] H. J. Patrick, A. D. Kersey, and F. Bucholtz, "Analysis of the response of long period fiber gratings to external index of refraction," IEEE J. Lightwave Technol., vol. 16, no. 9, pp. 1606-161, Sep. 1998.

[41] H. Tsuda, and K. Urabe, "Characterization of long-period grating refractive index sensors and their applications," Sensors, vol. 9, pp. 4559-4571, 2009.

[42] R. M. Waxler, and G. W. Cleek, "Refractive indices of fused silica at low temperatures," J. Res. Nat. Bur. Stand (Phys. And Chem.), vol. 75A, no. 4, pp. 279-281, Jul. 1971

[43] X. A. Aduriz, C. Lupi, N. Boyard, J. L. Bailleul, D. Leduc, V. Sobotka, N. Lefe'vre, X. Chapeleau, C. Boisrobert, and D. Delaunay, "Quantitative control of RTM6 epoxy resin polymerisation by optical index determination," Compos. Sci. Technol., vol. 67, no. 15, pp. 31963201, Dec. 2007.

Rebecca Y. N. Wong received her $\mathrm{PhD}$ in Engineering Photonics from Cranfield University, UK, in 2015. She is currently working at the Institute for Infocomm Research, Singapore. Her current research involves using optical fibre grating sensors and specialty fibres.

Edmon Chehura received a $\mathrm{PhD}$ in optical flow measurement instrumentation from Cranfield University, UK, in 2003. His current research interests are in optical fibre sensors and instrumentation. His work in this area encompasses the development and practical application of sensors for the measurement of a range of physical and chemical parameters. $\mathrm{He}$ is a member of the Institute of Physics, UK.

Stephen W. James gained an M.Sc. in 1988 in Applied Optics from Imperial College, London and a Ph.D. from the University of Southampton in 1992. He joined Cranfield University as a research fellow in 1993, where he developed his interest in the development and application of optical instrumentation and sensors. He leads the optical fiber sensing activity in the Centre of Engineering Photonics, with a focus on sensing chemical and physical parameters, and on deploying instrumentation in real world environments, ranging from foundation piles, railways, and aerospace structures to superconducting magnets. He is currently a Professor in the Centre of Engineering Photonics at Cranfield University. 
Ralph P. Tatam was appointed to a personal Chair in Engineering Photonics at Cranfield University in 1998. He graduated from Exeter University in 1981 (B.Sc. Physics and Chemistry) and was awarded a Ph.D. in Physics in 1986. He served a 5-year term as the European Editor for the journal Measurement Science and Technology and served as the Associate Editor for the SPIE journal Optical Engineering for 5 years. He has published over 450 papers in the field of optical instrumentation and sensors, and won the UK National
Measurement Awards Frontier Science and Measurement category in 2001 and 2004 for his work on planar Doppler velocimetry and nanostructured films on optical fibers, respectively. In 2005 he was awarded a D.Sc. by Exeter University and elected as a Fellow of the SPIE. 
2017-08-21

Resin directional flow and degree of cure sensing using chirped optical fibre long period gratings

Wong, Rebecca Y. N.

IEEE

RY Wong, E Chehura, S James, R Tatam. Resin directional flow and degree of cure sensing using chirped optical fibre long period gratings. IEEE Sensors Journal, Vol. 17, Issue 20, October 2017, pp. 6605-6614

http://dx.doi.org/10.1109/JSEN.2017.2739422

Downloaded from Cranfield Library Services E-Repository 